\title{
Finding critical exponents for two-dimensional Hamiltonian maps
}

\author{
Juliano A. de Oliveira, R. A. Bizão, and Edson D. Leonel \\ Departamento de Estatística, Matemática Aplicada e Computação, Instituto de Geociências e Ciências Exatas, Universidade Estadual \\ Paulista, Av. 24A, 1515 Bela Vista, CEP 13506-700 Rio Claro, SP, Brazil
}

(Received 30 September 2009; revised manuscript received 14 January 2010; published 26 April 2010)

\begin{abstract}
The transition from integrability to nonintegrability for a set of two-dimensional Hamiltonian mappings exhibiting mixed phase space is considered. The phase space of such mappings show a large chaotic sea surrounding Kolmogorov-Arnold-Moser islands and limited by a set of invariant tori. The description of the phase transition is made by the use of scaling functions for average quantities of the mapping averaged along the chaotic sea. The critical exponents are obtained via extensive numerical simulations. Given the mappings considered are parametrized by an exponent $\gamma$ in one of the dynamical variables, the critical exponents that characterize the scaling functions are obtained for many different values of $\gamma$. Therefore classes of universality are defined.
\end{abstract}

DOI: 10.1103/PhysRevE.81.046212

PACS number(s): 05.45.Ac, 05.45.Pq, 05.45.Tp

\section{INTRODUCTION}

Two-dimensional, nonlinear, area-preserving mappings have been considered in the study of dynamical systems for many years. Applications of this formalism can be used in study of channel flows, waveguide, transport properties, Fermi acceleration and also for the study of magnetic field lines in toroidal plasma devices with reversed shear (such as tokamaks) [1-10].

Generally, such kind of mappings have one or more control parameters. They can control the intensity of the nonlinearity, controlling also a transition from integrability to nonintegrability. The phase space of these mappings exhibit quite often mixed forms. One can observe a large chaotic sea that eventually surrounds Kolmogorov-Arnold-Moser islands and is limited by a set of invariant tori. The size of the chaotic sea is strongly influenced by the control parameters since they play the rule of controlling the intensity of the nonlinearity.

In this paper, we address specifically a transition from integrability to nonintegrability present in a special class of two-dimensional, nonlinear, area-preserving mappings. We look at average properties along the chaotic sea and therefore we find critical exponents that describe the behavior of average quantities of the chaotic sea. Such quantities are described in terms of scaling arguments, leading to a generic and robust description of a scaling observed for the chaotic sea. The critical exponents are obtained for several values of the control parameters. The knowledge of such exponents allow us to define and compare classes of universality for the two-dimensional Hamiltonian mappings.

The paper is organized as follows. In Sec. II we present the model and discuss the variables and control parameters used. Some properties of the phase space are also discussed, including a characterization of fixed points. The chaotic component of the phase space is characterized by Lyapunov exponents. In Sec. III we obtain and describe the critical exponents that define classes of universality. A merger of all the curves of the average quantity is obtained. Finally in the Sec. IV we present our concluding remarks.

\section{DEFINITION OF THE PROBLEM AND THE MAPPING}

In this section, we present and discuss some dynamical properties for a set of two-dimensional Hamiltonian mappings. We assume that there is a two-dimensional integrable system that is slightly perturbed. The Hamiltonian function that, in principle, describes the system is written as [11]

$$
H\left(I_{1}, I_{2}, \theta_{1}, \theta_{2}\right)=H_{0}\left(I_{1}, I_{2}\right)+\varepsilon H_{1}\left(I_{1}, I_{2}, \theta_{1}, \theta_{2}\right),
$$

where the variables $I_{i}$ and $\theta_{i}$ with $i=1,2$ correspond, respectively, to the action and angle. One can see clearly that the control parameter $\varepsilon$ controls a transition from integrability to nonintegrability. To use the characterization of the dynamics in terms of a mapping, we can now consider a Poincaré section defined by the plane $I_{1} \times \theta_{1}$ and assume $\theta_{2}$ as constant $(\bmod 2 \pi)$. A generic two-dimensional mapping which qualitatively describes the behavior of Eq. (1) is

$$
T:\left\{\begin{array}{l}
I_{n+1}=I_{n}+\varepsilon h\left(\theta_{n}, I_{n+1}\right) \\
\theta_{n+1}=\left[\theta_{n}+K\left(I_{n+1}\right)+\varepsilon p\left(\theta_{n}, I_{n+1}\right)\right] \bmod (2 \pi),
\end{array}\right.
$$

where $h, K$, and $p$ are assumed to be nonlinear functions of their variables while the index $n$ corresponds to the $n$th iteration of the mapping. The variables $I$ and $\theta$ correspond indeed to $I_{1}$ and $\theta_{1}$.

Since the mapping (2) should be area preserving, the expressions for $h\left(\theta_{n}, I_{n+1}\right)$ and $p\left(\theta_{n}, I_{n+1}\right)$ have to obey some properties, in particular some intrinsic relations. The relations are obtained considering that the determinant of the Jacobian matrix is the unity. After some straightforward algebra, it is easy to conclude that area preservation will be observed only if the condition

$$
\frac{\partial p\left(\theta_{n}, I_{n+1}\right)}{\partial \theta_{n}}+\frac{\partial h\left(\theta_{n}, I_{n+1}\right)}{\partial I_{n+1}}=0,
$$

is matched. For many mappings considered in the literature, the function $p\left(\theta_{n}, I_{n+1}\right)=0$. Hence, if we keep $h$ as $h\left(\theta_{n}\right)=\sin \left(\theta_{n}\right)$, and vary $K$, to illustrate applicability of the formalism, we nominate the following mappings that have already been studied:

(i) Considering $K\left(I_{n+1}\right)=I_{n+1}+\zeta I_{n+1}^{2}$, the logistic twist mapping is obtained [12]; 
(ii) $K\left(I_{n+1}\right)=I_{n+1}$, then the Taylor-Chirikov's map is recovered [13];

(iii) $K\left(I_{n+1}\right)=2 / I_{n+1}$, then the Fermi-Ulam accelerator model is obtained $[14,15]$;

(iv) $K\left(I_{n+1}\right)=\zeta I_{n+1}$, with $\zeta$ constant, then the bouncer model is found [16];

(v) For the case of

$$
K\left(I_{n+1}\right)= \begin{cases}4 \zeta^{2}\left(I_{n+1}-\sqrt{I_{n+1}^{2}-\frac{1}{\zeta^{2}}}\right) & \text { if } I_{n+1}>\frac{1}{\zeta}, \\ 4 \zeta^{2} I_{n+1} & \text { if } I_{n+1} \leq \frac{1}{\zeta} .\end{cases}
$$

where $\zeta$ is a constant, then we recovered the so called Hybrid-Fermi-Ulam-bouncer model [17].

In this paper, we consider the following expression for the two-dimensional mapping

$$
T:\left\{\begin{array}{c}
x_{n+1}=\left[x_{n}+\frac{a}{y_{n+1}^{\gamma}}\right] \bmod 1 \\
y_{n+1}=\left|y_{n}-b \sin \left(2 \pi x_{n}\right)\right|
\end{array},\right.
$$

where $a, b$, and $\gamma$ are the control parameters. The determinant of the Jacobian matrix is Det $\mathrm{J}=\operatorname{sign}\left[y_{n}-b \sin \left(2 \pi x_{n}\right)\right]$ where $\operatorname{sign}(u)=1$ if $u>0$ and $\operatorname{sign}(u)=-1$ if $u<0$. Note that if $\gamma<0$, depending on the initial conditions and control parameters, one can observe unlimited growth for the variable $\bar{y}$. Such growth is observed since large values of $y$ implies in a large number of oscillations for the sine function. Then, in the regime of very large oscillations, the sin function works more likely a random function yielding in an unlimited growth for $\bar{y}$. Since we want avoid such a condition in order to use the scaling approach, in this paper we consider only values for $0<\gamma \leq 1$.

Note however if we choose $\gamma=1$ and change the dynamical variables to $x \rightarrow v$ and $y \rightarrow \phi$, the mapping (5) recovers the one-dimensional Fermi-Ulam accelerator model where $v$ represents the velocity of the particle and $\phi$ denotes the phase of the moving wall. For this case the critical exponents are well defined [18].

It is important to emphasize that there are two control parameters in mapping (5) that control the transition from integrability to no integrability, namely, $a=0$ or $b=0$. The phase space generated from iteration of the mapping (5) for $a=2, b=10^{-3}$ and $\gamma=1 / 2$ is shown in Fig. 1 .

The fixed points of the mapping (5) can be obtained by matching the following conditions: $y_{n+1}=y_{n}=y$ and $x_{n+1}=x_{n}=x+m$, where $m=1,2,3, \ldots$ It is shown in Table I the fixed points obtained for different values of the exponent $\gamma$ and a fixed $a=2$, as well as their classification. We stress that fixed point 1, as labeled in Table I, is always hyperbolic, while fixed point 2 can be elliptic or hyperbolic, according to the combination of control parameters. Some of the elliptic fixed points are shown in Fig. 1 as (red) circles while some of the hyperbolic are identified as (blue) squares. Let us now discuss the characterization of the chaotic component of the phase space using Lyapunov exponents. Basically the procedure considers in evolving two neighboring initial conditions and check whether they diverge exponentially from each

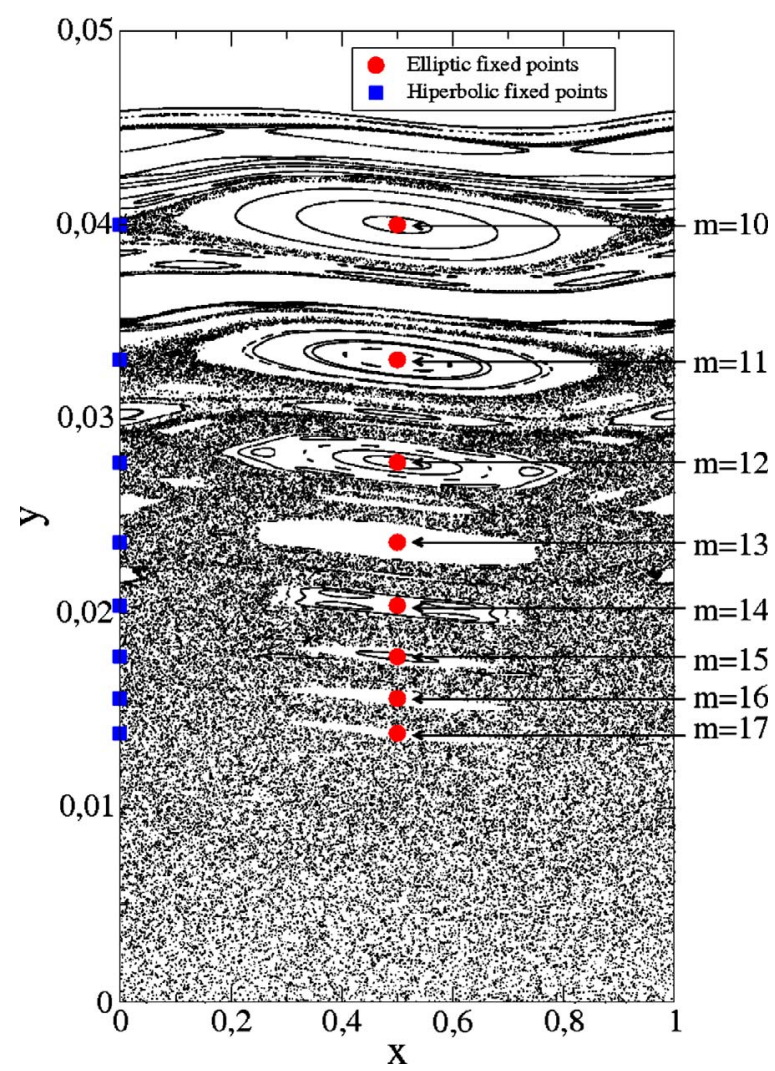

FIG. 1. (Color online) Phase space generated by the mapping Eq. (5) for the control parameters, $a=2, b=10^{-3}$, and $\gamma=1 / 2$. The (red) circle bullets indicate the location of some of the elliptic fixed points, each one of them enumerated, while the (blue) square furnish the position of some of the hyperbolic fixed points. Their numeration follow the same scheme, at the same value of $y$, of the elliptic fixed points.

other, as time evolves, or not. If the Lyapunov exponent is positive, say $\lambda>0$, the system has a chaotic component.

The Lyapunov exponents are defined as [19]

$$
\lambda_{j}=\lim _{n \rightarrow \infty} \frac{1}{n} \ln \left|\Lambda_{j}^{(n)}\right|, \quad j=1,2,
$$

where $\Lambda_{j}^{(n)}$ are the eigenvalues of the matrix $M=\prod_{i=1}^{n} J_{i}(x, y)$ where $J_{i}$ is the Jacobian matrix of the mapping evaluated along the orbit.

TABLE I. Fixed points and their classification for different values of $\gamma$.

\begin{tabular}{ccccc}
\hline \hline$\gamma$ & Fixed point 1 & Fixed point 2 & Elliptic & Hyperbolic \\
\hline$\frac{2}{3}$ & {$\left[0,\left(\frac{a}{m}\right)^{3 / 2}\right]$} & {$\left[\frac{1}{2},\left(\frac{a}{m}\right)^{3 / 2}\right]$} & $0<m<\left(\frac{62^{19 / 78}}{b \pi}\right)^{2 / 5}$ & $m>\left(\frac{62^{19 / 7}}{b \pi}\right)^{2 / 5}$ \\
$\frac{2}{5}$ & {$\left[0,\left(\frac{a}{m}\right)^{5 / 2}\right]$} & {$\left[\frac{1}{2},\left(\frac{a}{m}\right)^{5 / 2}\right]$} & $0<m<\left(\frac{20 \sqrt{2}}{b \pi}\right)^{2 / 5}$ & $m>\left(\frac{20 \sqrt{2}}{b \pi}\right)^{2 / 5}$ \\
$\frac{1}{2}$ & {$\left[0,\left(\frac{a}{m}\right)^{2}\right]$} & {$\left[\frac{1}{2},\left(\frac{a}{m}\right)^{2}\right]$} & $0<m<\left(\frac{16}{b \pi}\right)^{1 / 3}$ & $m>\left(\frac{16}{b \pi}\right)^{1 / 3}$ \\
$\frac{3}{4}$ & {$\left[0,\left(\frac{a}{m}\right)^{4 / 3}\right]$} & {$\left[\frac{1}{2},\left(\frac{a}{m}\right)^{4 / 3}\right]$} & $0<m<\left(\frac{162^{1 / 3}}{3 b \pi}\right)^{1 / 2}$ & $m>\left(\frac{162^{1 / 3}}{3 b \pi}\right)^{1 / 2}$ \\
$\frac{3}{5}$ & {$\left[0,\left(\frac{a}{m}\right)^{5 / 3}\right]$} & {$\left[\frac{1}{2},\left(\frac{a}{m}\right)^{5 / 3}\right]$} & $0<m<\left(\frac{202^{2 / 3}}{3 b \pi}\right)^{3 / 8}$ & $m>\left(\frac{202^{2 / 3}}{3 b \pi}\right)^{3 / 8}$ \\
$\frac{4}{5}$ & {$\left[0,\left(\frac{a}{m}\right)^{5 / 4}\right]$} & {$\left[\frac{1}{2},\left(\frac{a}{m}\right)^{5 / 4}\right]$} & $0<m<\left(\frac{52^{1 / 4}}{b \pi}\right)^{4 / 9}$ & $m>\left(\frac{52^{1 / 4}}{b \pi}\right)^{4 / 9}$ \\
1 & $\left(0, \frac{a}{m}\right)$ & $\left(\frac{1}{2}, \frac{a}{m}\right)$ & $0<m<\left(\frac{4}{b \pi}\right)^{1 / 2}$ & $m>\left(\frac{4}{b \pi}\right)^{1 / 2}$ \\
\hline \hline
\end{tabular}



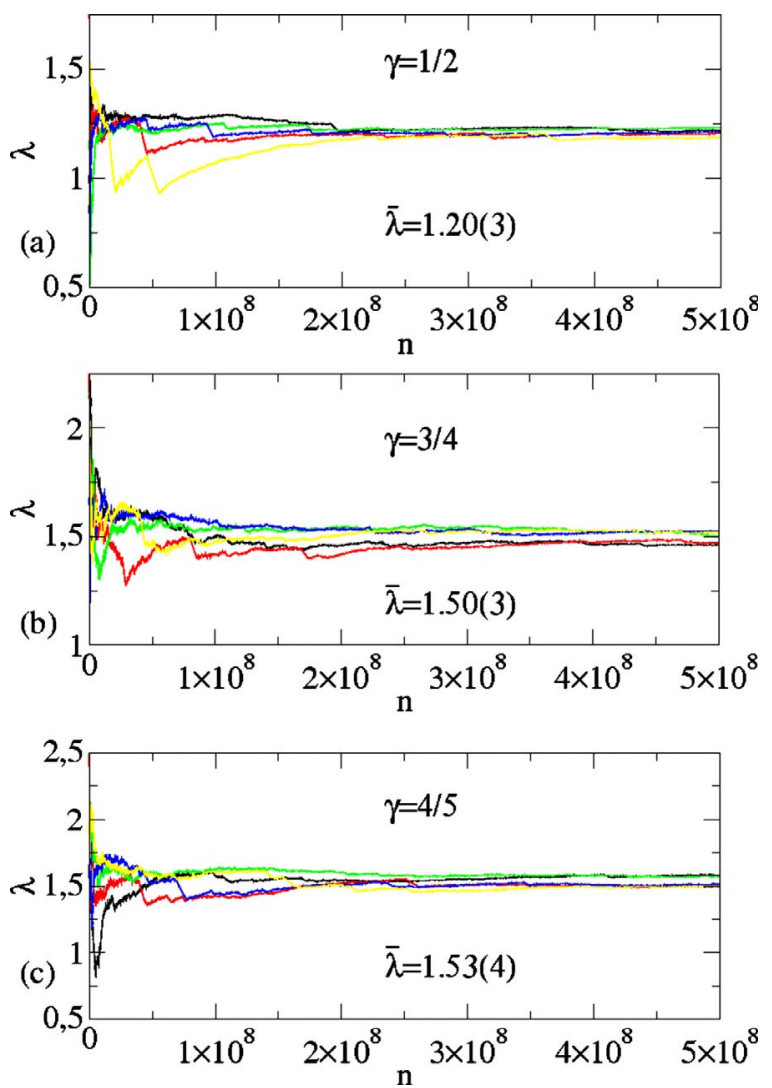

FIG. 2. (Color online) Plot of the positive Lyapunov exponents for the control parameters $a=2$ and $b=10^{-4}$ and (a) $\gamma=1 / 2$; (b) $\gamma=3 / 4$; and (c) $\gamma=4 / 5$.

It is shown in Fig. 2, the behavior of the positive Lyapunov exponent averaged along the chaotic sea for the mapping (5). The control parameters used were $a=2, b=10^{-4}$ and three different values of $\gamma$, namely: (a) $\gamma=1 / 2$; (b) $\gamma=3 / 4$; and (c) $\gamma=4 / 5$, as labeled in the figure. We have used an ensemble of five randomly chosen initial conditions along the chaotic sea and each initial condition was iterated up to $5 \times 10^{8}$ times. The average value obtained for $\gamma=1 / 2$ was $\bar{\lambda}=1.20(3)$ where the error 0.03 corresponds the deviation of the five sample. Considering $\gamma=3 / 4$ we found $\bar{\lambda}=1.50$ (3), while for the case of $\gamma=4 / 5$ we obtained $\bar{\lambda}=1.53(4)$.

We now discuss the behavior of the Lyapunov exponents as function of the parameters $a, b$, and $\gamma$. It is shown in Fig. 3(a) the behavior of $\bar{\lambda} \times b$ for a fixed $a=2$ and $\gamma=3 / 4$. Despite the variation in the control parameter for a large range of $b \in\left[10^{-5}, 10^{-3}\right]$, the positive Lyapunov exponent, shown in a $\log \times \operatorname{linear}$ plot, does not exhibit a representative variation. A similar behavior was observed in Fig. 3(b) for the case of $\bar{\lambda} \times a$ for a fixed $b=10^{-2}$ and same $\gamma$ used in Fig. 3(a) and a large range of $a \in\left[10,10^{2}\right]$. The variation in the control parameter $\gamma$ produces a change in the Lyapunov exponent, as shown in Fig. 3(c) for $\bar{\lambda} \times \gamma$. The control parameters considered in Fig. 3(c) were $a=10$ and $b=10^{-2}$ while $\gamma \in[0.1,1]$. For this case, the positive Lyapunov exponent grows linearly with the control parameter $\gamma$. A linear fit furnishes $\bar{\lambda}=0.71(1)+1.00(2) \gamma$.
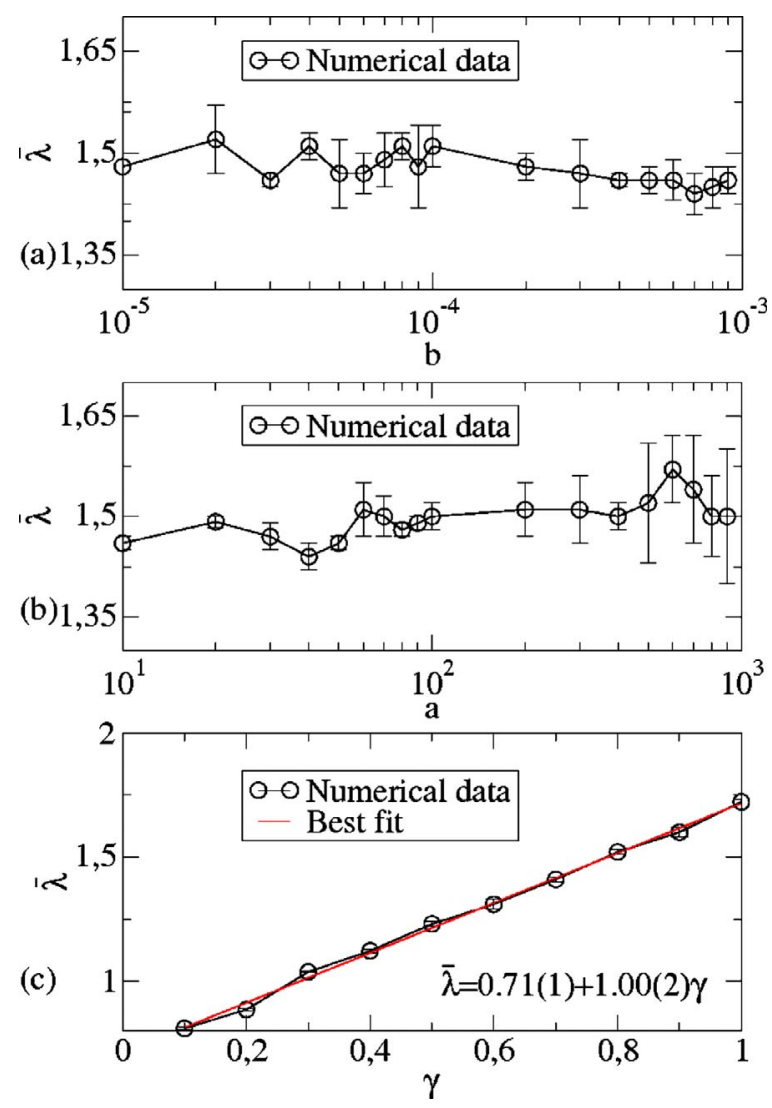

FIG. 3. (Color online) Plot of the average Lyapunov exponents for the control parameters: (a) $\gamma=3 / 4$ and $a=2$; (b) $\gamma=3 / 4$ and $b=10^{-2}$; and (c) $a=10$ and $b=10^{-2}$.

\section{NUMERICAL RESULTS AND SCALING PROPERTIES}

In this section, we concentrate to discuss some scaling properties present in the chaotic sea. The average quantity to be explored is the deviation of the average $\bar{y}$ for chaotic orbits, denoted as $\omega$. In fairness, the behavior of $\omega$ shows the same properties of the average $\bar{y}$. It is defined as

$$
\omega(n, a, b)=\frac{1}{M} \sum_{i=1}^{M} \sqrt{y_{i}^{2}(n, a, b)-\bar{y}_{i}^{2}(n, a, b)},
$$

where $M$ corresponds to an ensemble of different initial conditions $x_{i} \in(0,1)$ randomly chosen for a fixed $y_{0}=10^{-3} b$ and $\bar{y}_{i}$ is given by

$$
\overline{y_{i}}(n, a, b)=\frac{1}{n} \sum_{j=1}^{n} y_{j, i} .
$$

The behavior of $\omega \times n$ for different control parameters, as labeled in the figure, is shown in Fig. 4. However, similar results would indeed be observed for other values of $\gamma$ too. We have considered fixed the exponent $\gamma=3 / 4$.

Let us now discuss the behavior observed in Fig. 4. The curves start growing for small $n$ and after reaching a critical crossover iteration number, $n_{x}$, they bend toward a regime of convergence. Based on the behavior seen in Fig. 4(a) we can suppose that 

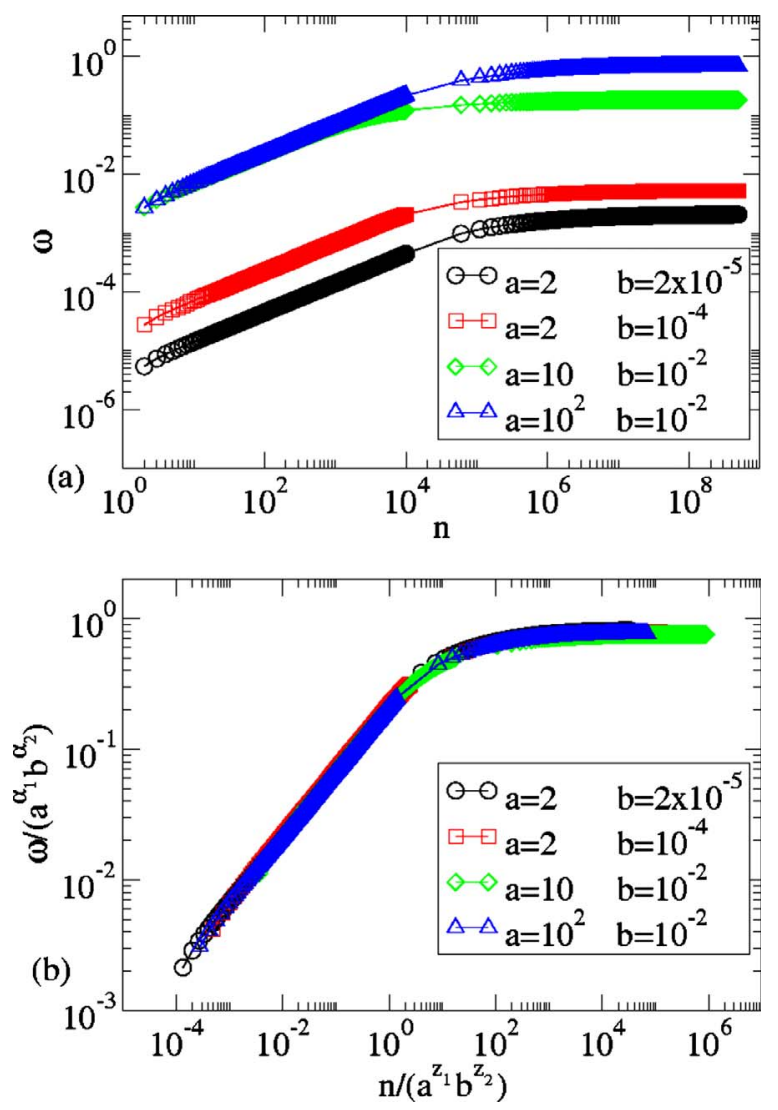

FIG. 4. (Color online) (a) Plot of different $\omega$ curves as function of $n$ for the control parameters $\gamma=3 / 4$, and different values of $a$ and $b$ for an ensemble of $M=5000$ different initial conditions. (b) Their collapse onto a single and universal plot.

(i) For $n \ll n_{x}, \omega$ grows according to a power law of the type

$$
\omega \propto\left(n b^{2}\right)^{\beta},
$$

where $\beta$ is a critical exponent;

(ii) For large $n$, say $n \gg n_{x}$, the behavior of $\omega$ is

$$
\omega \propto a^{\alpha_{1}} b^{\alpha_{2}}
$$

where $\alpha_{1}$ and $\alpha_{2}$ are critical exponents;

(iii) The crossover $n_{x}$, that characterizes the transition of the growing regime for the saturation is

$$
n_{x} b^{2} \propto a^{z_{1}} b^{z_{2}}
$$

where $z_{1}$ and $z_{2}$ are called as dynamical exponents.

The critical exponents $\alpha_{1}, \alpha_{2}$ and the dynamical exponents $z_{1}$ and $z_{2}$ can be obtained from extensive numerical simulations. First, fitting the initial regime of growth, we obtain that the critical $\beta \cong 0.5$. The other critical exponents are obtained from specific plots. It is shown in Figs. 5(a) and 5 (b) a plot $n_{x} \times b$ and $\omega_{\text {sat }} \times b$ respectively for the case where $a=2$. On the other hand, Figs. 5(c) and 5(d) plots $n_{x} \times a$ and $\omega_{\text {sat }} \times a$ for the case of a fixed control parameter $b=10^{-2}$. All the results shown in the Fig. 5 were obtained for a fixed $\gamma=3 / 4$ and using very long simulations of $5 \times 10^{8}$ iterations. The ensemble average used was $M=5 \times 10^{3}$.
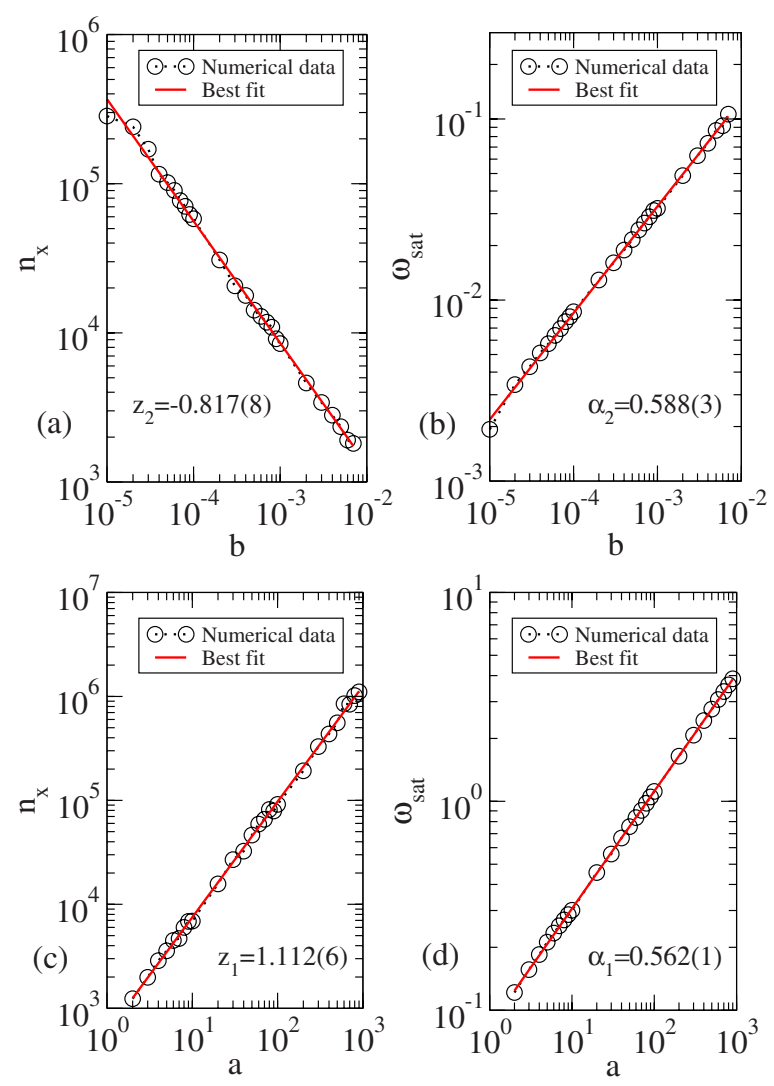

FIG. 5. (Color online) Plot for the case of the fixed control parameter $\gamma=3 / 4$. The parameters used were: (a) $a=2$ for $n_{x} \times b$, (b) $a=2$ for $\omega_{\text {sat }} \times b$, (c) $b=10^{-2}$ for $n_{x} \times a$, and (d) $b=10^{-2}$ and $\omega_{\text {sat }} \times a$.

A power law fitting to the curves plotted in Fig. 5 furnishes that: (a) $z_{2}=-0.817(8)$ and (b) $\alpha_{2}=0.588(3)$, while (c) $z_{1}=1.112(6)$ and (d) $\alpha_{1}=0.562(1)$. Since we have now obtained the critical exponents, the scaling hypotheses can be verified. In this case, it is shown in Fig. 4(b) a merger of four different curves of $\omega$ generated for different values of the control parameters $a$ and $b$ into a single and universal plot.

All the curves of $\omega$ presented above were obtained for a fixed $\gamma=3 / 4$. The procedure does indeed works well for other values of $\gamma$ too. It is shown in Table II the critical exponents found via extensive numerical simulations. Table II was obtained for a fixed control parameter $a=2$. However, if we keep as fixed the control parameter $b$, the critical exponents are shown in Table III. We stress that all curves obtained for different control parameters shall experience the same merger if the axis are rescaled properly. The behavior shown in both the Tables II and III can be presented in terms of a plot, as shown in Fig. 6.

One can see in Fig. 6 that as the control parameter $\gamma$ rises, there is a decrease in both the exponents $\alpha_{1}$ and $\alpha_{2}$. Since $\alpha_{i}$, $i=1,2$ are related to the behavior of $\omega$ in the saturation regime, i.e., for sufficiently long $n$, such a decrease implies that for a large value of $\gamma$, the saturation will be achieved at lower values than those compared to the same control parameters but for smaller $\gamma$. The behavior of the crossover $n_{x}$ is also strongly affected since the critical exponents $z_{i}, i=1,2$ are modeled by decreasing functions of $\gamma$. 
TABLE II. Critical exponents obtained for $a=2$ and $b \in\left[10^{-5}, 10^{-3}\right]$ for the mapping (5).

\begin{tabular}{ccll}
\hline \hline$\gamma$ & \multicolumn{1}{c}{$\beta$} & \multicolumn{1}{c}{$\alpha_{2}$} & \multicolumn{1}{c}{$z_{2}$} \\
\hline $2 / 5$ & $0.481(5)$ & $0.75(1)$ & $-0.56(1)$ \\
$3 / 7$ & $0.487(7)$ & $0.696(4)$ & $-0.57(2)$ \\
$4 / 9$ & $0.484(5)$ & $0.710(2)$ & $-0.58(1)$ \\
$1 / 2$ & $0.488(4)$ & $0.673(2)$ & $-0.641(7)$ \\
$3 / 5$ & $0.488(5)$ & $0.59(1)$ & $-0.68(2)$ \\
$2 / 3$ & $0.489(7)$ & $0.607(1)$ & $-0.757(4)$ \\
$5 / 7$ & $0.491(5)$ & $0.5893(9)$ & $-0.808(5)$ \\
$3 / 4$ & $0.488(6)$ & $0.588(3)$ & $-0.817(8)$ \\
$4 / 5$ & $0.489(7)$ & $0.563(1)$ & $-0.858(6)$ \\
1 & $0.495(6)$ & $0.518(4)$ & -1 \\
\hline \hline
\end{tabular}

Let us now discuss about the classes of universality. First we have to stress that the phase transition we are considering in this paper is not defined in the rigorous Statistical Mechanics point of view. The transition we are taking into account describes the behavior of average quantities, along the chaotic sea, as function of the control parameters which control the intensity of the nonlinearity of the mappings. Moreover, the critical exponents we found for the average quantities are written as function of the control parameters. After finding these critical exponents, we can rescale the system conveniently so that, after a reparametrization, all quantities are scale independent. The set of critical exponents obtained define which class of universality the system belongs to. Therefore, to illustrate three cases, we consider the following examples: (i) Fermi-Ulam model and periodically corrugated waveguide; (ii) a dissipative bouncer model and a stochastic version of the Fermi-Ulam model with the particle experiencing inelastic collisions; (iii) a classical particle confined in an infinitely deep potential well containing an oscillating square well [20] or time modulated barrier [21] and mapping (4) with $\gamma=1 / 2$. These three cases illustrate two different phase transitions.

TABLE III. Critical exponents obtained for the range $a \in\left[10,10^{2}\right]$ and $b=10^{-2}$ for the mapping (5).

\begin{tabular}{cccc}
\hline \hline$\gamma$ & $\beta$ & \multicolumn{1}{c}{$\alpha_{1}$} & \multicolumn{1}{c}{$z_{1}$} \\
\hline $1 / 5$ & $0.489(6)$ & $0.820(2)$ & $1.64(1)$ \\
$1 / 4$ & $0.491(5)$ & $0.791(1)$ & $1.57(1)$ \\
$1 / 3$ & $0.489(5)$ & $0.741(1)$ & $1.464(7)$ \\
$2 / 5$ & $0.492(6)$ & $0.704(1)$ & $1.38(1)$ \\
$3 / 7$ & $0.491(5)$ & $0.6929(9)$ & $1.34(1)$ \\
$4 / 9$ & $0.492(5)$ & $0.680(3)$ & $1.342(6)$ \\
$1 / 2$ & $0.492(5)$ & $0.659(1)$ & $1.33(1)$ \\
$3 / 5$ & $0.493(5)$ & $0.611(2)$ & $1.209(7)$ \\
$2 / 3$ & $0.491(6)$ & $0.587(1)$ & $1.162(5)$ \\
$3 / 4$ & $0.492(5)$ & $0.562(1)$ & $1.112(6)$ \\
$4 / 5$ & $0.492(5)$ & $0.547(1)$ & $1.088(5)$ \\
1 & $0.496(4)$ & $0.4967(4)$ & $0.987(4)$ \\
\hline \hline
\end{tabular}
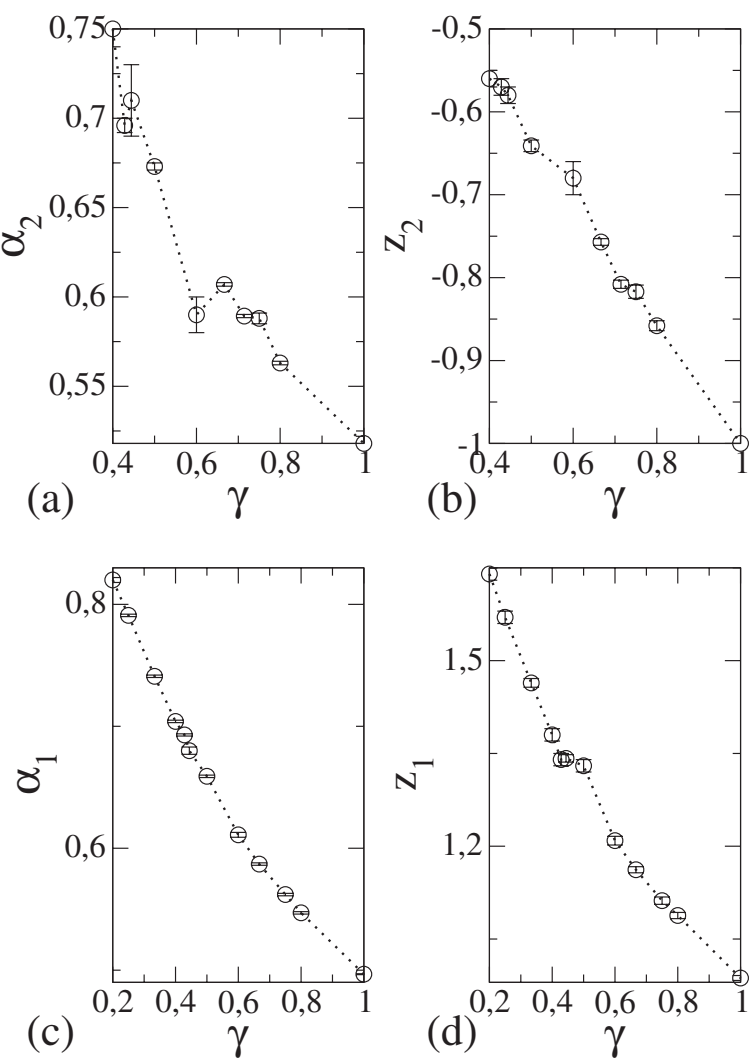

FIG. 6. Plot of the critical exponents as function of the parameter $\gamma$ : (a) $\alpha_{1} \times \gamma$; (b) $z_{1} \times \gamma$; (c) $\alpha_{2} \times \gamma$; and (d) $z_{2} \times \gamma$.

In the case (i), the Fermi-Ulam model (a classical particle confined to bounce between two walls; one of them is fixed and the other one is periodically time varying-collisions are elastic and no damping forces are present) is described via a two-dimensional, nonlinear, area-preserving mapping for the variables velocity of the particle and phase of the moving wall. The critical exponents obtained for the average velocity along the chaotic sea were [18]: $\alpha=0.5, \beta=0.5$, and $z=-1$. On the other hand, the corrugate waveguide model considers the description of a light-beam moving inside two mirrors where one of them is flat and the other one is periodically corrugated. The mapping describes the position of the light beam at each reflection with the mirror and the angle of the light-beam trajectory [22], then there is no velocity involved in this model. The critical exponents obtained were the same as those obtained for the Fermi-Ulam model. The models are totally independent from each other, but then, near such a transition, the chaotic sea has qualitatively the same general behavior, thus the two models belong to the same class of universality, near this transition.

For the case (ii), let us point out that the Fermi-Ulam model, in its deterministic version, does not have Fermi acceleration, thanks to the presence of a set of invariant spanning curves in the phase space. Fermi acceleration is a phenomenon where a classical particle can acquires unlimited energy after collisions with an infinitely heavy and moving wall. However, if one introduce a stochastic force on the moving wall, then Fermi acceleration is produced. Moreover, if we consider that the particle is suffering inelastic collisions 
with the boundary such collisions are sufficient conditions to suppress Fermi acceleration [23]. Notice however that the bouncer model (a classical particle falling in a constant gravitational field and hitting a time moving boundary) does indeed exhibit Fermi acceleration for some specific initial conditions and control parameters. If one introduce inelastic collisions with the moving wall, then Fermi acceleration is also suppressed [24,25]. The critical exponents for both cases are obtained as $\alpha=-0.5, \beta=0.5$, and $z=-1$. For this case, the transition considered was from limited to unlimited energy gain. Since the two sets of critical exponents are the same, then the two models belong to the same class of universality near such a phase transition.

In the last case (iii), the model consists of a classical particle confined to move inside an infinitely deep potential well which contains: (a) oscillating square well [20] or (b) oscillating potential barrier [21]. The dynamics of either cases is described by a two-dimensional area-preserving-map with more than one nonlinearity. The phase space is mixed and invariant tori prevent the unlimited energy gain. The chaotic sea of both models is described by scaling arguments leading to the following critical exponents: $\alpha \cong 2 / 3, \beta=0.5$, and $z \cong 4 / 3$. These exponents are the same as those obtained by us for $\gamma=1 / 2$ in mapping (4) (see Table III). Therefore, despite the differences of the models and the mapping, the critical exponents are the same, therefore the models belong to the same class of universality near this phase transition from integrability to non integrability.

Finally, the Tables II and III bring a set of critical exponents which could be used to define classes of universality and compared to other kinds of transition observed in dynamical systems.

\section{CONCLUSION}

To summarize our conclusions, we have studied in this work a phase transition from integrability for nonintegrability for a set two-dimensional Hamiltonian maps. The critical exponents were obtained via extensive simulations and scaling hypotheses were all supported by a perfect collapse of all the curves of the deviation around the average quantities for the chaotic sea. Lyapunov exponents have also been used to quantify the intensity of chaos of the mappings.

\section{ACKNOWLEDGMENTS}

J.A.O. and R.A.B. thank to CNPq. E.D.L. kindly acknowledges the financial support from CNPq, FAPESP, and FUNDUNESP, Brazilian agencies.
[1] G. A. Luna-Acosta, J. A. Méndez-Bermudéz, P. Seba, and K. N. Pichugin, Phys. Rev. E 65, 046605 (2002).

[2] G. M. Zaslavsky, Phys. Rep. 371, 461 (2002).

[3] D. G. Ladeira and J. K. L. da Silva, J. Phys. A: Math. Theor. 40, 11467 (2007).

[4] R. Venegeroles, Phys. Rev. Lett. 101, 054102 (2008); 99, 014101 (2007); 102, 064101 (2009).

[5] S. M. Soskin, R. Mannella, and O. M. Yevtushenko, Phys. Rev. E 77, 036221 (2008).

[6] F. R. N. Koch, F. Lenz, C. Petri, F. K. Diakonos, and P. Schmelcher, Phys. Rev. E 78, 056204 (2008).

[7] J. E. Howard, A. J. Lichtenberg, M. A. Lieberman, and R. H. Cohen, Physica D 20, 259 (1986).

[8] F. Saif, Phys. Lett. A 274, 98 (2000).

[9] F. Saif, I. Bialynicki-Birula, M. Fortunato, and W. P. Schleich, Phys. Rev. A 58, 4779 (1998).

[10] G. A. Luna-Acosta, K. Na, L. E. Reichl, and A. Krokhin, Phys. Rev. E 53, 3271 (1996).

[11] A. J. Lichtenberg and M. A. Lieberman, Regular and Chaotic Dynamics (Springer Verlag, New York, 1992).

[12] J. E. Howard and J. Humpherys, Physica D 80, 256 (1995).
[13] B. V. Chirikov, Phys. Rep. 52, 263 (1979).

[14] M. A. Lieberman and A. J. Lichtenberg, Phys. Rev. A 5, 1852 (1972).

[15] J. K. L. da Silva, D. G. Ladeira, E. D. Leonel, P. V. E. McClintock, and S. O. Kamphorst, Braz. J. Phys. 36, 700 (2006).

[16] L. D. Pustylnikov, Trans. Mosc. Math. Soc. 2, 1 (1978).

[17] E. D. Leonel and P. V. E. McClintock, J. Phys. A 38, 823 (2005).

[18] E. D. Leonel, P. V. E. McClintock, and J. K. L. da Silva, Phys. Rev. Lett. 93, 014101 (2004).

[19] J. P. Eckmann and D. Ruelle, Rev. Mod. Phys. 57, 617 (1985).

[20] E. D. Leonel and P. V. E. McClintock, Chaos 15, 033701 (2005).

[21] E. D. Leonel and P. V. E. McClintock, Phys. Rev. E 70, 016214 (2004).

[22] E. D. Leonel, Phys. Rev. Lett. 98, 114102 (2007).

[23] E. D. Leonel, J. Phys. A: Math. Theor. 40, F1077 (2007).

[24] E. D. Leonel and A. L. P. Livorati, Physica A 387, 1155 (2008).

[25] Andre Luis Prando Livorati, D. G. Ladeira, and E. D. Leonel, Phys. Rev. E 78, 056205 (2008). 